\title{
Deployment of COVID-19 diagnostic testing: challenges and key considerations for the future
}

\author{
Martin Möckel ${ }^{1}$, Fausto Baldanti ${ }^{2}$, Nirmal K Ganguly ${ }^{3}$, Guiqiang Wang ${ }^{4}$, Luke A O’Neil ${ }^{5}$, \\ Harald Renz ${ }^{6}$, Carlos Eduardo Dos Santos Ferreira ${ }^{7}$, Kazuhiro Tateda ${ }^{8}$, and Barbara Van \\ Der $\mathrm{Pol}^{9}$ \\ ${ }^{1}$ Charité -Universitätsmedizin \\ ${ }^{2}$ Diagnostic and Pediatric Sciences, University of Pavia \\ ${ }^{3}$ 2National Institute of Immunology, New Delhi, India and Institute of Liver and Biliary \\ Science, New Delhi, India and Policy Center for Biomedical Research, Translational Health \\ Science \& Technology Institute, Faridabad, Haryana, India \\ ${ }^{4}$ The Center for Liver Diseases, Peking University First Hospital \\ ${ }^{5}$ Trinity Biomedical Sciences Institute, Trinity College Dublin \\ ${ }^{6}$ Institute of Laboratory Medicine and Pathobiochemistry, Molecular Diagnostics Philipps, \\ University Marburg University Hospital Giessen and Marburg GmbH \\ ${ }^{7}$ Albert Einstein Hospital \\ ${ }^{8}$ Toho University \\ ${ }^{9}$ University of Alabama at Birmingham
}

April 2, 2021

\section{Introduction}

Since the outbreak of COVID-19 there has been an unprecedented effort from the scientific community to develop tools to help tackle this crisis. According to the Foundation for Innovative New Diagnostics (FIND; 18 March 2021) there are 1025 commercialized SARS-CoV-2 assays currently available and an additional 98 in development, including 655 immunoassays and 437 molecular assays. ${ }^{1}$ Early in the pandemic, molecular testing using nucleic acid amplification tests (NAATs) became the pillar of COVID-19 diagnostics. Since then, the development of antigen tests, immunoassays, point-of-care, and centralized options means there are now choices to be made as to how, when, and where to deploy these technologies and many guidelines have been developed, often based on Centers for Disease Control and Prevention (CDC) and World Health Organization (WHO) guidance. ${ }^{2-4}$ However, with the rapid emergence of new technologies and new scientific data, these guidelines are very fluid and subject to change. Furthermore, while NAATs are the most sensitive diagnostic tool for COVID-19 infection, ${ }^{3-5}$ the global demand for diagnostic testing is still such that the use of labour-intensive, specialist techniques needs to be carefully considered.

The aim of this review is to provide an international evaluation of real-world testing needs and to define: settings where the 'next best' alternatives to NAATs are appropriate; settings where NAATs may not be the best option; how to manage antigen test results; and how to manage negative NAAT results where there is still a strong clinical suspicion of COVID-19. We will further aim to set out the key considerations for defining a testing strategy. Table 1 demonstrates that each testing strategy provides different information on infection status and has different performance metrics, so the right option for the right setting needs to 
be carefully assessed. Here we also discuss the common challenges facing clinicians and laboratorians when interpreting and supplying COVID-19 diagnostics and provide insights into what will be needed next.

Table 1. A summary of the diagnostic testing methodologies for COVID-19.

\begin{tabular}{|c|c|c|c|c|c|}
\hline & Measure & Platforms/techr & $\begin{array}{l}\text { Turnaround } \\
\text { otiogies(range) }\end{array}$ & $\begin{array}{l}\text { Number of } \\
\text { samples per } \\
\text { run/test }\end{array}$ & $\begin{array}{l}\text { Performance } \\
\text { range LOD } \\
\text { sensitiv- } \\
\text { ity/specificity } \\
(\%)\end{array}$ \\
\hline \multirow{6}{*}{$\begin{array}{l}\text { NAATs for } \\
\text { viral RNA } \\
\text { antigen } \\
\text { detection (NP } \\
\text { swab, } \\
\text { oropharyngeal } \\
\text { swab, sputum, } \\
\text { bronchoalveolar } \\
\text { lavage fluid, } \\
\text { others) }\end{array}$} & $\begin{array}{l}\text { Direct } \\
\text { detection of } \\
\text { SARS-CoV-2 } \\
\text { viral RNA }\end{array}$ & $\begin{array}{l}\text { High throughput } \\
\text { RT-PCR }\end{array}$ & $1.5-8$ hours & Up to 384 & $\begin{array}{l}>1.23 \mathrm{cp} / \mu \mathrm{L}^{7} \\
450-540,000 \\
\mathrm{NDU} / \mathrm{mL}^{8} \\
>90 \% / \mathrm{up} \text { to } \\
100 \%\end{array}$ \\
\hline & & $\begin{array}{l}\text { Point-of-care } \\
\text { RT-PCR }\end{array}$ & 20 mins & 1 & $>12 \mathrm{cp} / \mathrm{mL}^{7}$ \\
\hline & & $\begin{array}{l}\text { High- } \\
\text { throughput } \\
\text { TMA }\end{array}$ & 3 hours & Unconfirmed & $600 \mathrm{NDU} / \mathrm{mL}^{9}$ \\
\hline & & $\begin{array}{l}\text { Point-of-care } \\
\text { LAMP }\end{array}$ & $20-60$ mins & 1 & $\begin{array}{l}>10 \mathrm{cp} / \mu \mathrm{l}^{7} \\
>75 \% \\
\text { sensitivity }{ }^{10}\end{array}$ \\
\hline & & $\begin{array}{l}\text { High- } \\
\text { throughput } \\
\text { LAMP } \\
\text { (fluorescence) }\end{array}$ & 45 mins & 96 & $>1 \mathrm{cp} / \mu \mathrm{l}$ \\
\hline & & $\begin{array}{l}\text { CRISPR/LAMP } \\
\text { lateral flow }\end{array}$ & 15 mins & 1 & $>6.75 \mathrm{cp} / \mu \mathrm{L}^{7}$ \\
\hline $\begin{array}{l}\text { Antigen } \\
\text { detection } \\
\text { (saliva, NP } \\
\text { swab) }\end{array}$ & $\begin{array}{l}\text { Immunoassays } \\
\text { for the } \\
\text { detection of } \\
\text { SARS-CoV-2 } \\
\text { viral antigens }\end{array}$ & $\begin{array}{l}\text { High- } \\
\text { throughput } \\
\text { centralized }\end{array}$ & From 18 mins & Up to 300 & $\begin{array}{l}\text { Sensitivity } \\
(95 \% \mathrm{CI})<5 \\
\text { days post } \\
\text { symptom } \\
\text { onset and Ct } \\
<30: 97.5 \% \\
(92.8-99.5 \%) \\
\mathrm{Ct}>30: 26.7 \% \\
(12.3-45.9 \%)^{11}\end{array}$ \\
\hline
\end{tabular}




\begin{tabular}{|c|c|c|c|c|c|}
\hline & Measure & Platforms/techr & $\begin{array}{l}\text { Turnaround } \\
\text { otiogies(range) }\end{array}$ & $\begin{array}{l}\text { Number of } \\
\text { samples per } \\
\text { run/test }\end{array}$ & $\begin{array}{l}\text { Performance } \\
\text { range LOD } \\
\text { sensitiv- } \\
\text { ity/specificity } \\
(\%)\end{array}$ \\
\hline \multirow{3}{*}{$\begin{array}{l}\text { Antibody } \\
\text { detection } \\
\text { (serum, plasma) }\end{array}$} & \multirow{3}{*}{$\begin{array}{l}\text { Detection of } \\
\text { immune } \\
\text { response i.e. } \\
\text { past exposure to } \\
\text { SARS-CoV-2 }\end{array}$} & $\begin{array}{l}\text { Point-of-care } \\
\text { (lateral flow) }\end{array}$ & $15-30$ mins & 1 & $\begin{array}{l}\text { Sensitivity (95\% } \\
\text { CI): } 28.9 \% \\
(16.4-44.3)- \\
98.3 \% \\
(91.1-99.7) \\
\text { Specificity (95\% } \\
\text { CI): } 92.4 \% \\
(87.4-95.9) \\
-100 \% \\
(99.7-100)^{12}\end{array}$ \\
\hline & & $\begin{array}{l}\text { High-throughput } \\
\text { centralized }\end{array}$ & $\begin{array}{l}\text { First results } \\
\text { from } 18 \text { mins to } \\
24 \text { hours }\end{array}$ & Up to 500 & $\begin{array}{l}\text { Typically, }>90 \% \\
\text { sensitive and } \\
>95 \% \text { specific }^{13}\end{array}$ \\
\hline & & $\begin{array}{l}\text { Point-of-care } \\
\text { (lateral flow) }\end{array}$ & 15 mins & 1 & $\begin{array}{l}\text { Typically, } \\
>90 \% \\
\text { sensitive and } \\
>95 \% \\
\text { specific }^{13}\end{array}$ \\
\hline
\end{tabular}

CI, confidence interval; cp, copies; CRISPR, clustered regularly interspaced short palindromic repeats; Ct, cycle threshold; LAMP, isothermal loop-mediated amplification; NAAT, nucleic acid amplification test; NDU, NAAT detectable units; NP, nasal pharyngeal; RNA, ribonucleic acid; RT-PCR, reverse transcription polymerase chain reaction; TMA, transcription-mediated amplification.

\subsection{Available classes of diagnostic tests}

In September 2020, the WHO set out their target product profiles for COVID-19 diagnostics, stating that only NAATs are recommended for confirmation of COVID-19 disease. ${ }^{14}$ Most NAATs have been based on reverse transcription polymerase chain reaction (RT-PCR), which enables highly sensitive and specific detection of viral RNA by targeting specific viral genes and amplifying the signal..$^{8,14,15}$ Transcription-mediated amplification (TMA) is another technique used interchangeably with RT-PCR that involves the isothermal amplification of RNA by reverse transcription and subsequent generation of numerous transcripts by RNA polymerase. ${ }^{16}$ Loop-mediated isothermal amplification (LAMP) is a NAAT which utilizes an isothermal reaction that does not require the thermocycling process of RT-PCR. ${ }^{17-19}$ Studies indicate that the LAMP technique is as highly specific as RT-PCR-based technologies but reports of sensitivities vary, with some studies reporting low sensitivity kits being marketed to developing countries. ${ }^{19-22}$ However, LAMP can be performed with minimal equipment and has been deployed to supplement widescale testing and/or where resources are limited..$^{23}$

In cases where NAATs are unavailable, turnaround times are unacceptably slow, or near-patient NAATs are necessary, rapid antigen tests may facilitate earlier diagnosis. ${ }^{14,19}$ Antigen tests are typically immunoassays designed to detect SARS-CoV-2 proteins and require no amplification. As a result, these assays often require 
less instrumentation and can be performed rapidly, often in near-patient settings rather than laboratories. ${ }^{24,25}$ This class of tests may allow patients to self-sample and supports high-throughput testing. ${ }^{24-27}$ However, antigen tests offer reduced sensitivity compared with NAATs, so adoption of these tests needs to be appropriate to the needs of the patient population served or the defined use-case (e.g. screening for same-day travel). ${ }^{28}$

Whilst NAATs are capable of diagnosing current infection, antibody testing identifies exposure to the pathogen over the patient's lifetime, supporting diagnosis later during the disease course. ${ }^{29}$ Antibody testing aids our understanding of COVID-19 and our immune response, ${ }^{30-33}$ the spread of infection, ${ }^{14,34-36}$ and, more recently, our response to vaccine administration and long-term efficacy. ${ }^{37}$

\section{Different testing settings}

In this review, we will focus our discussion on 7 key testing settings: symptomatic individuals presenting for diagnostic testing and/or treatment of COVID-19 symptoms; asymptomatic individuals accessing healthcare for planned non-COVID-19-related reasons; patients needing to access emergency care (symptom status unknown); patients being discharged from healthcare following hospitalization for COVID-19; healthy individuals in both single event settings (e.g. airports, restaurants, hotels, concerts, sporting events) and repeat access settings (e.g. workplaces, schools, universities); and vaccinated individuals. These 7 settings comprise the key areas where testing is being used to care for patients and help to prevent the spread of infection, and echoes the list provided by the WHO. ${ }^{3}$

Before diagnostic testing is considered in any individual, it is important to establish if they have symptoms and, if so, the time from symptom onset. ${ }^{2,4,5} \mathrm{On}$ an individual basis, these simple factors will be key to determining the relevant test choice as, for example, antigen testing may not be beneficial $>10$ days post symptom onset. ${ }^{38,39}$ 
Symptomatic individuals

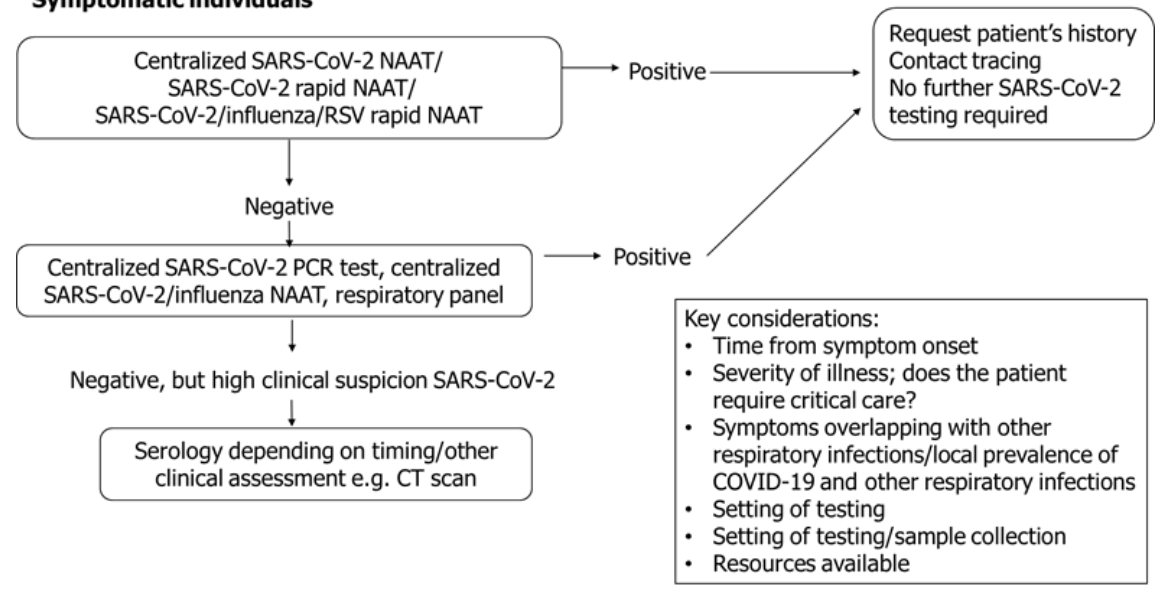

Asymptomatic individuals accessing healthcare

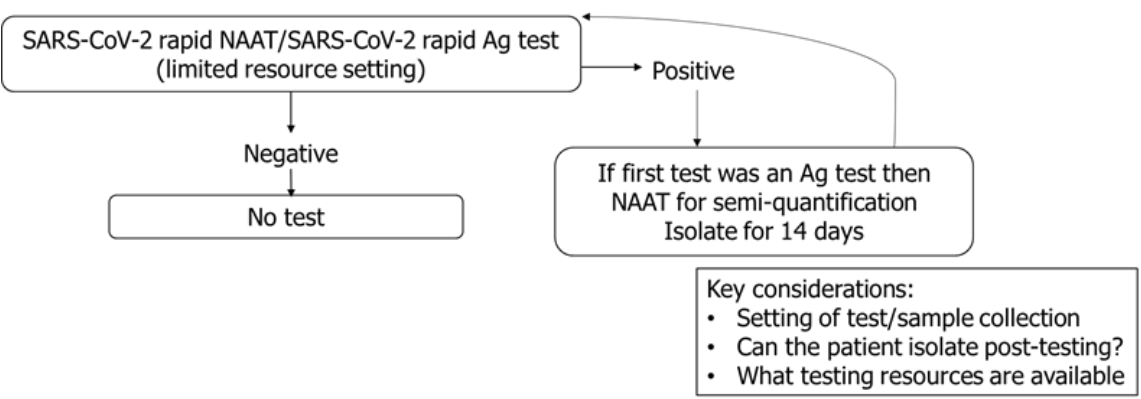

Discharge from healthcare

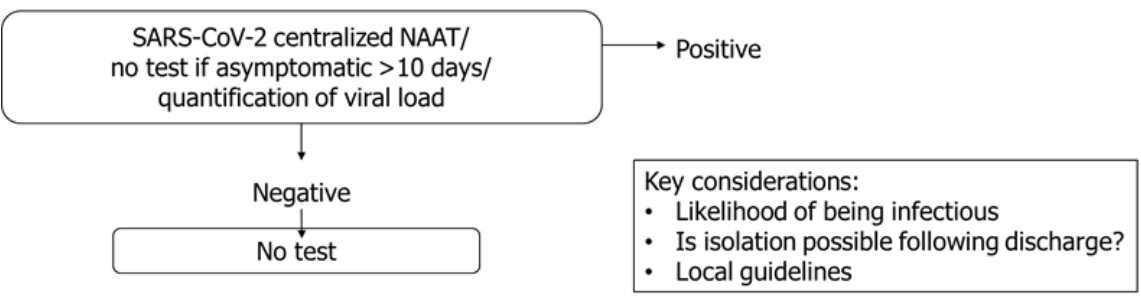

General population: single event

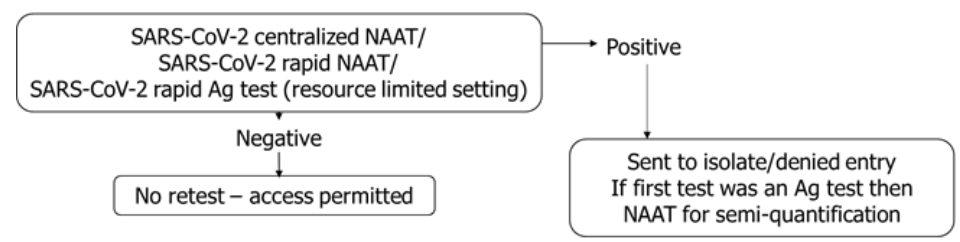

Key considerations:
- What timeline of testing prior to access is acceptable?
- What volume of testing is needed?
- Can samples be pooled?
- Lon testing be managed on-site?

General population: repeat access event

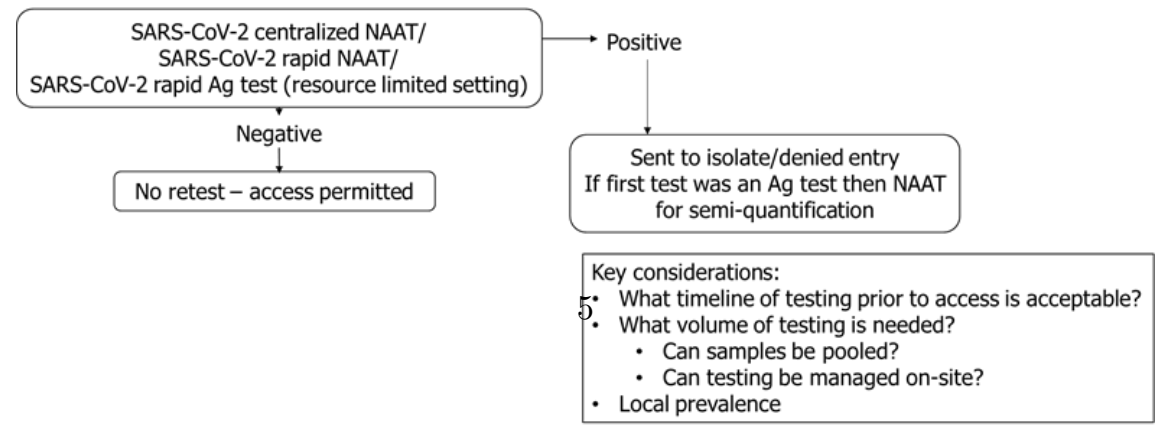


Figure 1. Testing strategies and considerations for the different diagnostic settings considered in this publication.

Ag, antigen; CT, computed tomography; NAAT, nucleic acid amplification test; PCR, polymerase chain reaction.

\section{Symptomatic patients}

\section{Current practices and guidelines/recommendations}

The priority for symptomatic patients is the need to know if their symptoms are due to COVID-19; in these cases, NAATs for COVID-19 should be performed when possible (Figure 1). NAATs are the most sensitive class of tests available, and this method will help to ensure that cases are not missed among symptomatic patients. ${ }^{26,40}$ In this context we are talking about RT-PCR testing and TMA, not LAMP, as sensitivity data are still variable (Table 1). ${ }^{17-20,23,41-43}$ In settings where testing resources are available but limited, many laboratories have adopted sample pooling strategies that allow conservation of resources. ${ }^{44}$ Several commercially available assays have regulatory authorization for pooling and offer guidance regarding the optimal number of samples to pool and the volume per sample to include in the pool ${ }^{45,46}$ The methods and benefits of pooling are highly influenced by the prevalence in the population being tested. For example, creating pools of 10 samples in a population of $10 \%$ positivity would require repeat testing of all of the individual samples in most pools, thus resulting in extra testing and an extended time to results. Conversely, pooling only 3 samples in a population with $1 \%$ positivity does not realize all of the reagent savings possible. Pooling strategies must be evaluated at each laboratory based on the population(s) they serve and can even be applied to sub-groups of samples sent to the laboratory to minimize time to results and maximize reagent conservation. ${ }^{47-49}$

In settings where NAATs are unavailable, antigen testing is also acceptable for the diagnosis of symptomatic patients as an option that is more informative than no testing. Antigen tests detect viral proteins in a patient's serum or plasma, and whilst they have a lower sensitivity than NAATs, they are most sensitive when viral loads are high, which may correlate with infectivity. ${ }^{50}$ If symptoms are strongly indicative of COVID19, a negative test should also be confirmed with a NAAT. ${ }^{51-54}$ The authors consider that specificity is not an issue with currently available antigen tests, and that whilst retesting is not needed to confirm positivity, NAATs may be performed to provide semi-quantitative cycle threshold $(\mathrm{Ct})$ values to aid understanding of infection status. ${ }^{26,28,52,54-56}$ The utility of $\mathrm{Ct}$ values is currently unclear and the use of $\mathrm{Ct}$ values to assess infection status is currently only deployed in certain regions, and only then in patients who require medical intervention for COVID-19.

Depending on the local prevalence and patient-specific risk of influenza, dual-target NAATs for influenza and COVID-19 may be useful for differential diagnosis, particularly if an initial NAAT result is negative and clinical suspicion of respiratory infection is high (Figure 1). However, in many regions the prevalence of influenza is very low, possibly due to infection control measures for COVID-19, and the risk of influenza is lower than the normal risk expected for many regional flu seasons. ${ }^{57-61}$

If a patient repeatedly tests negative, but their clinical presentation is highly suggestive of COVID-19 and a diagnosis is required to enable medical care, a low-dose chest-computed tomography (CT) scan could be used to diagnose or rule out COVID-19 ${ }^{62-64}$ However, this is recommended with caution, as chest-CT scans are less sensitive than NAATs for COVID-19, and specificity is often over-estimated due to selection bias and the low prevalence of other pulmonary disease in retrospective studies. The data suggest that chest-CT scans can be used to complement diagnostic testing but are not an effective standalone assessment. ${ }^{62,63}$ 


\section{Key considerations}

The key determinants of the test for use in symptomatic patients include the patient's symptoms/clinical presentation; whether the patient needs to be admitted for their symptoms or can manage their symptoms at home with isolation; and in the setting where patients are accessing testing/sampling and presenting to the healthcare system. ${ }^{65}$ Globally, there are vast differences in how and where symptomatic individuals access healthcare, such as walk-in/fever clinics, drive-through testing centers, at-home testing squads, postal testing, and in the hospital/emergency department (ED)/general (not COVID-specific) clinic/COVID-specific clinic. If patients are accessing testing in a setting where they could possibly pass infection on to others, strict hygiene measures need to be applied and sample collection needs to be done as quickly as possible. If patients are well enough not to require urgent admission, then centralized testing is acceptable. However, if patients need urgent medical care for their symptoms, then rapid testing at the point of care is required. Several NAATs have been developed that can be performed in near-patient settings and, if available and affordable, these offer advantages over antigen-based assays. In symptomatic individuals, test sensitivity is important to ensure that infectious individuals are not missed and do not continue to spread their infection, whilst also ensuring that those who need medical care are appropriately triaged.

\section{Asymptomatic hospital admissions}

Patients in this category would need testing prior to hospital admission in order to prevent nosocomial COVID-19 transmission and to triage patients appropriately within the hospital setting. ${ }^{66,67}$ These patients would not have symptoms of COVID-19 and would be attending routine healthcare appointments or planned surgeries; these appointments are not considered as urgent care.

\section{Current practices}

Point-of-care or centralized NAATs are generally used to test patients prior to admission. Patients may be able to isolate whilst they await their test results, making centralized testing possible in some settings. The urgency of the care that is required also determines whether the patient should have a rapid point-of-care test or whether a centralized option would be acceptable. As patients are asymptomatic there is no need for repeat testing; if they test positive, they should isolate for 14 days and then retest for COVID-19. ${ }^{67}$ As these patients are asymptomatic, antigen tests may not be adequately sensitive to detect COVID-19 (Table 1). ${ }^{4,53,68}$ In this setting, if patients do have a positive antigen test result they should be treated similarly to patients with a positive NAAT, requiring them to isolate for 14 days and retest (Figure 1).

\section{Key considerations}

NAATs are the most sensitive method for detection of SARS-CoV-2 and would maximally prevent the spread of infection to the healthcare system. A disadvantage of using NAATs in this setting is that studies have shown prolonged NAAT positivity in patients who are no longer symptomatic following infection, and it may be that non-infectious virus is detected. ${ }^{50,69,70} 71$

Antigen testing is not as sensitive as a NAAT, particularly in asymptomatic individuals, and as such, there is the possibility that false-negative tests may leave the healthcare system exposed to infection. ${ }^{72}$ However, this risk is currently mitigated by the universal COVID-19 precautions utilized in healthcare settings and will be further mitigated once the healthcare workforce is vaccinated. Antigen tests would be more useful if they could reliably indicate whether or not a patient is infectious, preventing unnecessary hospitalization. More information regarding how diagnostics tests relate to infectivity is needed before this is possible. 


\section{Urgent hospital admission, asymptomatic/symptom status un- known}

\section{Current practices}

When patients need critical care, point-of-care NAATs will provide swift and accurate results (Figure 1). These patients are not being admitted due to symptoms of COVID-19 and, as such, repeat testing following a negative NAAT test would not be required, unless indicators arise to suggest a patient does have respiratory symptoms.

\section{Key considerations}

If results can be obtained more quickly using an antigen test in this clinical setting, then an antigen test in the interim is also acceptable; however, the result should be confirmed with a NAAT, which might be performed on site in a centralized laboratory. ${ }^{66}$ In patients with respiratory symptoms in the ED setting, antigen testing has still been shown to produce false-negative results. ${ }^{66}$ Depending on the clinical setting and the care that the patient requires, other assessments for the presenting condition may also reveal the likelihood of a respiratory infection but are not diagnostic for COVID-19. ${ }^{73}$

\section{Discharge from healthcare following treatment for COVID-19}

\section{Current practices}

Globally, the approach to managing patients leaving healthcare following COVID-19 is variable. In the US and Japan, patients are expected to be symptom free for a period of at least 10 days; in Germany, patients must have a $\mathrm{Ct}$ value $<30$ for discharge to nursing homes; in Italy, the absence of symptoms and a negative NAAT are the requirements for discharge. ${ }^{74-76}$ Other countries only require a negative NAAT if the patient was severely unwell (e.g. receiving supportive oxygen). ${ }^{74}$ In China, patients are discharged if they are no longer symptomatic and have a repeated negative NAAT for COVID-19 within 24 hours. ${ }^{74}$ Chinese patients are then required to isolate for a further 14 days and may be discharged to an interim/recovery hospital for further isolation and monitoring before returning home. ${ }^{70}$ These stringent criteria are due to reports of relapsing infection and aim to prevent transmissions in these cases. ${ }^{77}$

\section{Key considerations}

Patients are tested prior to discharge to ensure they are not infectious. Particular patient populations, such as post-transplant and/or immunocompromised patients, stay PCR-positive for a longer time period. ${ }^{78} \mathrm{Ct}$ values can provide a guide to infection status - a recovered patient with a high Ct corresponds with a low viral load, where a patient is unlikely to be infectious. ${ }^{79,80}$ However, Ct values need to be standardized as there is variability between platforms, between labs, and between reagent lots within a single lab. ${ }^{79}$ As such, they can provide clinical guidance only once the standardization practices for estimating viral concentration from $\mathrm{Ct}$ values is in place. $\mathrm{Ct}$ values are not routinely standardized against viral concentration ranges and, additionally, not all NAATs provide a Ct, meaning Ct values currently have limited clinical utility. ${ }^{8079} \mathrm{In}$ the future, quantification of viral load and standardization of Ct values may be widely applicable, aiding determination of infectious periods and possibly reducing the duration of hospitalization for some patient populations. In patients who remain NAAT-positive for a prolonged time period, antigen testing may better reflect if a patient is still infectious, as previously described above. 


\section{The general population}

\section{Single event}

Here we define a 'single event' as an event that will not be routinely repeated with the same group of individuals (e.g. accessing an airport for travel, visiting a restaurant, or visiting a stadium as a spectator). This category encompasses a broad range of scenarios and recommendations will need to be specific for each setting.

\section{Single event current practices}

There is no standardized approach to testing within the community, both NAATs and antigen tests are being used. As an example scenario, many airlines and airports are developing pre-flight testing requirements, and currently these have not been regulated by the international community or even by local governments. ${ }^{81}$

When considering the appropriate test, if individuals can isolate between their test and the access event, then centralized NAATs could be used to deliver high volumes (e.g. people accessing an airport or a stadium event). In this asymptomatic population, pooling samples could be useful in order to maximize the testing capacity, although this may reduce the sensitivity of tests and, as such, is most appropriate for use with centralized PCR testing as the most sensitive method of detection. ${ }^{44,47-49,82}$ However, this would not be practical for activities such as visiting a shopping center or restaurant. Highly sensitive antigen or NAAT point-of-care tests could be performed by non-laboratory trained personnel in these settings; however, whilst these tests provide quick results (10-15 minutes), they may not be suitable to conduct in crowded environments. Lateral flow antigen tests could be a simple and cost-effective way to test large groups of people; however, studies report high numbers of false-negative and false-positive results, meaning COVID-19 precautions should still be employed. ${ }^{28,83,84}$

\section{Single event key considerations}

When considering testing of the general asymptomatic population for access to single events, the key considerations should include:

- Population issues (e.g. if the population are asymptomatic). What is the local prevalence of infection? Are people attending from higher prevalence regions? This is very important as testing the general population may result in a high number of false-positive results ${ }^{83}$

- Sensitivity requirements for this population and the potential impact of a false-negative result. Is it necessary to detect all infected individuals, or only anyone highly infectious? Is catching the 'most infectious' cases sufficient? Will other measures, such as mask wearing and social distancing, be possible in this setting?

- The feasibility of testing at scale. What scale of testing is possible in that setting? Is reliable testing feasible? If self-swabs are used, how is the quality checked and will swabbing be supervised? What is the prevalence of infection?

\section{Repeat access}

Repeat access settings comprise workplaces, universities, schools, and hospitals, where the same group of people repeatedly interact together.

\section{Repeat access current practices}

Testing in repeat access settings is already being widely conducted, for example, many hospitals are regularly testing their healthcare staff using NAATs. ${ }^{85}$ In healthcare staff, regular testing is leading to the identification 
of many COVID-19 cases, enabling prompt isolation and therefore limiting outbreaks within hospitals. ${ }^{86}$ In healthcare settings, testing staff has clear benefits for the costs involved with screening: the prevention of COVID-19 outbreaks among hospital staff, subsequent staff absences, and nosocomial transmission to potentially vulnerable patients. Pooling samples can also help to make screening these populations more resource efficient. ${ }^{44,82}$

Professional athletes and their support staff around the world have been subject to regular testing so that sports can continue during the pandemic. This is often supported by isolation, social distancing, personal protective equipment for staff, and other measures to prevent infection. ${ }^{87-89}$ In these professional settings, the funding is often available to test regularly to ensure that the sports continue to operate, and these decisions sit with the sporting leagues and national governments.

For schools and universities, NAATs may not be needed, as antigen testing or LAMP may be sufficient to detect the most infectious cases. In addition, older students may be able to adhere to some social distancing and mask wearing measures. Overall, transmission has been noted to be lower in younger pupils compared with older pupils. ${ }^{90,91}$ Whilst the cost-benefit of NAATs for screening in school students would likely prove inappropriate, antigen testing may be suitable. Pooling NAAT samples could also help to manage NAAT testing volumes; however, this should be approached cautiously as methods are not standardized and pooling techniques are not automated, which does compromise efficiency. ${ }^{44}$

In these repeat access settings, a single infection could become an outbreak. Importantly, local prevalence of infection and necessity of the contact should be carefully considered before allowing any gathering of individuals.

\section{Repeat access key considerations}

Similarly to single access events, the prevalence of infection is an important consideration when sampling is being carried out, as well as the frequency of testing. In Germany, healthcare workers are currently tested on a weekly basis using a NAAT; however, testing frequency will need to be determined on the basis of each situation, the risk of infection, and the cost-benefit.

\section{Vaccinated individuals}

Several COVID-19 vaccines have now been shown to provide protection against COVID-19 and many more are still under development. ${ }^{92,93}$ There are still many unanswered questions regarding the longevity of immunity offered by vaccines, if they will be efficacious against all strains of COVID-19, which vaccines are most efficacious in different patient cohorts, and if vaccinated individuals are able to acquire and transmit COVID19 without becoming infected. Studies are ongoing to answer all these questions using a range of testing strategies.

In order to assess the longevity of vaccine-mediated immunity, high-throughput quantitative anti-spike (S) antibody tests (as most vaccines currently elicit a response against the SARS-CoV-2 S- antigen) will be useful. ${ }^{94}$ Anti-nucleocapsid antibody tests may also be used to assess natural versus vaccine-mediated immunity. ${ }^{95-97}$ Many serological assays have been shown to correlate with neutralizing antibody titres; ${ }^{98,99}$ however, direct assessment of neutralizing antibodies is preferable where possible as it is not fully understood how antibody test positivity relates to protective immunity against SARS-CoV-2. ${ }^{96,100}$ Assessments of cellular immunity are also necessary to completely understand how COVID-19 vaccines offer protection and how long this protection lasts.

In order to assess whether new variants are emerging that have the potential to escape vaccine-mediated immunity, full genome analysis is needed to better track evolution and spread of lineages, with particular focus on regular S gene sequence analysis and vaccinated sera challenge studies of emergent strains. ${ }^{101,102}$ 


\section{What are the testing considerations for the next steps in the pan- demic?}

A main focus for diagnostics will now be the ongoing monitoring of emergent strains. As discussed, this will be essential to ensure that the global rollout of vaccines is successful and to help the international community emerge from the pandemic. ${ }^{101}$ Currently, as most NAATs detect several SARS-CoV-2 genetic targets, it is considered unlikely that mutations will lead to false-negative results; however, the FDA have requested that laboratorians are mindful that this may occur. ${ }^{103}$

Standardization of Ct values or fully standardized quantitative NAATs for SARS-CoV-2 will be extremely useful to assess efficacy of interventions in COVID-19 patients, to help determine when individuals are safe to leave quarantine and staff are safe to return to work. ${ }^{104}$ Development of reference material to enable standardization of these assays is recommended.

The data regarding the relationship between diagnostic parameters and infectivity is currently limited, and research is ongoing in this area to clarify exactly how these parameters correlate. Recommendations on the most appropriate diagnostic test for different utilities may change once more information is available on these points.

The use of alternative sample types to the nasal pharyngeal (NP) swab, such as saliva, and the use of new technologies, such as CRISPR-based tests, are under investigation, and these may also provide different opportunities for testing and additional considerations (e.g. those concerning school populations or mass testing of large groups). ${ }^{30,43,105,106}$ Self-sampling methods that provide increased sensitivity could broaden the capacity for mass testing prior to events or entering the workplace. ${ }^{27} \mathrm{As}$ it will take time before vaccination will be able to eradicate COVID-19, testing measures will continue to be important.

\section{Acknowledgements}

Medical writing support was provided by Rose-Marie Falconer at Elements Communications and was funded by Roche Diagnostics.

\section{References}

1. FIND. COVID-19 pipeline. 2021. https://www.finddx.org/covid-19/pipeline/ (Accessed March 2021).

2. The European Centre For Disease Prevention and Control. COVID-19 Testing strategies and objectives. 2020. https://www.ecdc.europa.eu/en/covid-19/surveillance/testing-strategies (Accessed March 2021).

3. The World Health Organization. Diagnostic testing for SARS-CoV-2. 2020. https://www.who.int/publications/i/item/diagnostic-testing-for-sars-cov-2 (Accessed March 2021).

4. Arevalo-Rodriguez I, Seron P, Buitrago-Garcia D, et al. Recommendations for SARS-CoV-2/COVID-19 testing: a scoping review of current guidance. BMJ Open 2021; 11 (1): e043004.

5. The US Centers for Disease Prevention and Control. Testing for COVID-19. 2020. https://www.cdc.gov/coronavirus/2019-ncov/symptoms-testing/testing.html (Accessed March 2021).

6. Mostafa HH, Hardick J, Morehead E, Miller JA, Gaydos CA, Manabe YC. Comparison of the analytical sensitivity of seven commonly used commercial SARS-CoV-2 automated molecular assays. J Clin Virol2020; 130 : 104578.

7. Yu CY, Chan KG, Yean CY, Ang GY. Nucleic Acid-Based Diagnostic Tests for the Detection SARSCoV-2: An Update. Diagnostics (Basel)2021; 11 (1).

8. The US Food and Drug Administration. SARS-CoV-2 Reference Panel Comparative Data. 2020. https://www.fda.gov/medical-devices/coronavirus-covid-19-and-medical-devices/sars-cov-2-referencepanel-comparative-data (Accessed February 2021). 
9. Hologic. Hologic SARS-CoV-2 Assays. 2021. https://www.hologic.com/hologic-products/diagnosticsolutions/hologic-sars-cov-2-assays (Accessed March 2021).

10. Rodel J, Egerer R, Suleyman A, et al. Use of the variplex SARS-CoV-2 RT-LAMP as a rapid molecular assay to complement RT-PCR for COVID-19 diagnosis. J Clin Virol 2020; 132 : 104616.

11. Roche Diagnostics. Elecsys SARS-CoV-2 Antigen. 2021. https://diagnostics.roche.com/global/en/products/params/elecsys-sars-cov-2-antigen-test.html (Accessed March 2021).

12. Hayer J, Kasapic D, Zemmrich C. Real-world clinical performance of commercial SARS-CoV-2 rapid antigen tests in suspected COVID-19: A systematic meta-analysis of available data as per November 20, 2020. medRxiv 2020: 2020.12.22.20248614.

13. The US Food and Drug Administration. EUA Authorized Serology Test Performance. 2021. https://www.fda.gov/medical-devices/coronavirus-disease-2019-covid-19-emergency-useauthorizations-medical-devices/eua-authorized-serology-test-performance. (Accessed March 2021)

14. The World Health Organization. COVID-19 Target product profiles for priority diagnostics to support response to the COVID-19 pandemic v.1.0. 2020. https://www.who.int/publications/m/item/covid19-target-product-profiles-for-priority-diagnostics-to-support-response-to-the-covid-19-pandemic-v.0.1 (Accessed March 2021).

15. Wirden M, Feghoul L, Bertine M, et al. Multicenter comparison of the Cobas 6800 system with the RealStar RT-PCR kit for the detection of SARS-CoV-2. J Clin Virol 2020; 130 : 104573.

16. Gorzalski AJ, Tian H, Laverdure C, et al. High-Throughput Transcription-mediated amplification on the Hologic Panther is a highly sensitive method of detection for SARS-CoV-2. J Clin Virol 2020;129 : 104501 .

17. Fowler VL, Armson B, Gonzales JL, et al. A highly effective reverse-transcription loop-mediated isothermal amplification (RT-LAMP) assay for the rapid detection of SARS-CoV-2 infection. J Infect2021; 82 (1): $117-25$.

18. Mautner L, Baillie CK, Herold HM, et al. Rapid point-of-care detection of SARS-CoV-2 using reverse transcription loop-mediated isothermal amplification (RT-LAMP). Virol J 2020; 17 (1): 160.

19. Osterdahl MF, Lee KA, Lochlainn MN, et al. Detecting SARS-CoV-2 at point of care: preliminary data comparing loop-mediated isothermal amplification (LAMP) to polymerase chain reaction (PCR). $B M C$ Infect Dis 2020; 20 (1): 783.

20. Freire-Paspuel B, Garcia-Bereguiain MA. Low clinical performance of the Isopollo COVID-19 detection kit (M Monitor, South Korea) for RT-LAMP SARS-CoV-2 diagnosis: A call for action against low quality products for developing countries. Int J Infect Dis 2021; 104 : 303-5.

21. Iacobucci G. Covid-19: Mass population testing is rolled out in Liverpool. BMJ 2020; 371 : m4268.

22. Iacobucci G. Covid-19: Rapid test missed over 50\% of positive cases in Manchester pilot. BMJ 2020; $371: \mathrm{m} 4323$.

23. The UK Department of Health and Social Care. Clinical evaluation confirms accuracy of LAMP test. Gov.uk; 2020.

24. Lanser L, Bellmann-Weiler R, Ottl KW, et al. Evaluating the clinical utility and sensitivity of SARSCoV-2 antigen testing in relation to RT-PCR Ct values. Infection 2020.

25. Mattiuzzi C, Henry BM, Lippi G. Making sense of rapid antigen testing in severe acute respiratory syndrome coronavirus 2 (SARS-CoV-2) diagnostics. Diagnosis (Berl) 2020. 
26. Dinnes J, Deeks JJ, Adriano A, et al. Rapid, point-of-care antigen and molecular-based tests for diagnosis of SARS-CoV-2 infection. Cochrane Database Syst Rev 2020; 8 : CD013705.

27. Lindner AK, Nikolai O, Kausch F, et al. Head-to-head comparison of SARS-CoV-2 antigen-detecting rapid test with self-collected nasal swab versus professional-collected nasopharyngeal swab. Eur Respir $J 2021$.

28. Houston H, Gupta-Wright A, Toke-Bjolgerud E, Biggin-Lamming J, John L. Diagnostic accuracy and utility of SARS-CoV-2 antigen lateral flow assays in medical admissions with possible COVID-19. J Hosp Infect 2021.

29. Chen M, Qin R, Jiang M, Yang Z, Wen W, Li J. Clinical applications of detecting IgG, IgM or IgA antibody for the diagnosis of COVID-19: A meta-analysis and systematic review. Int $J$ Infect Dis 2021;104 : 415-22.

30. Pisanic N, Randad PR, Kruczynski K, et al. COVID-19 Serology at Population Scale: SARS-CoV-2Specific Antibody Responses in Saliva.J Clin Microbiol 2020; 59 (1).

31. Long QX, Liu BZ, Deng HJ, et al. Antibody responses to SARS-CoV-2 in patients with COVID-19. Nat Med 2020; 26 (6): 845-8.

32. Röltgen K, Powell AE, Wirz OF, et al. Defining the features and duration of antibody responses to SARS-CoV-2 infection associated with disease severity and outcome. Science Immunology 2020;5 (54): eabe0240.

33. Schaffner A, Risch L, Weber M, et al. Sustained SARS-CoV-2 nucleocapsid antibody levels in nonsevere COVID-19: a population-based study. Clin Chem Lab Med 2020; 59 (2): e49-e51.

34. Fujita K, Kada S, Kanai O, et al. Quantitative SARS-CoV-2 Antibody Screening of Healthcare Workers in the Southern Part of Kyoto City During the COVID-19 Pre-pandemic Period. Front Public Health2020; $8: 595348$.

35. Mahase E. Covid-19: Antibody prevalence in England fell from $6.0 \%$ to $4.4 \%$ over three months, study finds. BMJ 2020; 371 : $\mathrm{m} 4163$.

36. Vogl T, Leviatan S, Segal E. SARS-CoV-2 antibody testing for estimating COVID-19 prevalence in the population. Cell Rep Med2021: 100191.

37. Bradley T, Grundberg E, Selvarangan R. Antibody responses boosted in seropositive healthcare workers after single dose of SARS-CoV-2 mRNA vaccine. medRxiv 2021.

38. Mallett S, Allen AJ, Graziadio S, et al. At what times during infection is SARS-CoV-2 detectable and no longer detectable using RT-PCR-based tests? A systematic review of individual participant data. $B M C$ Medicine 2020; 18 (1): 346.

39. Sethuraman N, Jeremiah SS, Ryo A. Interpreting Diagnostic Tests for SARS-CoV-2. JAMA 2020; 323 (22): 2249-51.

40. Boger B, Fachi MM, Vilhena RO, Cobre AF, Tonin FS, Pontarolo R. Systematic review with metaanalysis of the accuracy of diagnostic tests for COVID-19. Am J Infect Control 2021; 49 (1): 21-9.

41. Gonzalez-Gonzalez E, Lara-Mayorga IM, Rodriguez-Sanchez IP, et al. Colorimetric loop-mediated isothermal amplification (LAMP) for cost-effective and quantitative detection of SARS-CoV-2: the change in color in LAMP-based assays quantitatively correlates with viral copy number. Anal Methods 2021; 13 (2): 169-78.

42. Huang WE, Lim B, Hsu CC, et al. RT-LAMP for rapid diagnosis of coronavirus SARS-CoV-2. Microb Biotechnol 2020; 13 (4): 950-61. 
43. Nagura-Ikeda M, Imai K, Tabata S, et al. Clinical Evaluation of Self-Collected Saliva by Quantitative Reverse Transcription-PCR (RT-qPCR), Direct RT-qPCR, Reverse Transcription-Loop-Mediated Isothermal Amplification, and a Rapid Antigen Test To Diagnose COVID-19.J Clin Microbiol 2020; 58 (9).

44. The US Centers for Disease Prevention and Control. COVID-19 Pooling Procedures. 2020. https://www.cdc.gov/coronavirus/2019-ncov/lab/pooling-procedures.html (Accessed March 2021).

45. Viracor. Emergency Use Authorisation (EUA) Summary Viracor SARS-CoV-2 Assay. 2021. https://www.fda.gov/media/143069/download (Accessed March 2021 2021).

46. Perkin Elmer. Instructions for PerkinElmer@ New Coronavirus Nucleic Acid Detection Kit 2020. https://www.fda.gov/media/136410/download (Accessed March 2021).

47. Public Health England. Standard operating procedure: Pooling of asymptomatic SARS-CoV2 COVID-19 sample for (PCR/or other) testing. 2020. https://www.england.nhs.uk/coronavirus/wpcontent/uploads/sites/52/2020/09/C0777-sample-pooling-sop-v1.pdf (Accessed February 2021).

48. Petrovan V, Vrajmasu V, Bucur AC, et al. Evaluation of Commercial qPCR Kits for Detection of SARSCoV-2 in Pooled Samples.Diagnostics (Basel) 2020; 10 (7).

49. Torres I, Albert E, Navarro D. Pooling of nasopharyngeal swab specimens for SARS-CoV-2 detection by RT-PCR. J Med Virol 2020;92 (11): 2306-7.

50. Guglielmi G. Fast corornavirus tests: what they can and can't do. Nature. 2020.

51. The US Food and Drug Administration. Coronavirus Disease 2019 Testing Basics. 2019. https://www.fda.gov/consumers/consumer-updates/coronavirus-disease-2019-testing-basics (Accessed February 2021).

52. The European Centre for Disease Control. Options fo the use of rapid antigen tests for COVID-19 in the EU/EEA and the UK. 2020. https://www.ecdc.europa.eu/sites/default/files/documents/Options-use-ofrapid-antigen-tests-for-COVID-19.pdf (Accessed February 2021).

53. The US Centers for Disease Prevention and Control. Using Antigen Tests. 2020. https://www.cdc.gov/coronavirus/2019-ncov/lab/resources/antigen-tests-guidelines.html (Accessed February 2021).

54. B-Fast Network of the National Research Network for COVID-19; Protzer UK, I; Renz, H; Busch, D; Denkinger, C; Fatkenheuer, G; Klein, F; Krausslich, H-G; Krefing, D; Schiethauer, S. Position paper of the B-FAST network in the National Research Network of University Medicine on COVID-19 on the application and approval practice of rapid antigen tests for the detection of the new coronavirus, SARS-CoV-2. 2021.

55. Carpenter CR. Rapid antigen and molecular tests had varied sensitivity and $>/=97 \%$ specificity for detecting SARS-CoV-2 infection. Ann Intern Med 2020; 173 (12): JC69.

56. Mak GC, Cheng PK, Lau SS, et al. Evaluation of rapid antigen test for detection of SARS-CoV-2 virus. J Clin Virol 2020;129 : 104500.

57. Chan CP, Wong NS, Leung CC, Lee SS. Positive impact of measures against COVID-19 on reducing influenza in the Northern Hemisphere.J Travel Med 2020; 27 (8).

58. Chow A, Hein AA, Kyaw WM. Unintended Consequence: Influenza plunges with public health response to COVID-19 in Singapore. J Infect2020; 81 (2): e68-e9.

59. Kuo SC, Shih SM, Chien LH, Hsiung CA. Collateral Benefit of COVID-19 Control Measures on Influenza Activity, Taiwan. Emerg Infect Dis2020; 26 (8): 1928-30.

60. Noh JY, Seong H, Yoon JG, Song JY, Cheong HJ, Kim WJ. Social Distancing against COVID-19: Implication for the Control of Influenza.J Korean Med Sci 2020; 35 (19): e182. 
61. Olsen SJ, Azziz-Baumgartner E, Budd AP, et al. Decreased Influenza Activity During the COVID-19 Pandemic - United States, Australia, Chile, and South Africa, 2020. MMWR Morb Mortal Wkly Rep 2020;69 (37): 1305-9.

62. Islam MN, Dipi RM, Mostafa SN, Datta A. Progression of Disease in COVID-19 Patients Evaluated by Chest CT Imgaing and Correlated with Clinical Parameters. Mymensingh Med J 2021; 30 (1): 182-8.

63. Waller JV, Kaur P, Tucker A, et al. Diagnostic Tools for Coronavirus Disease (COVID-19): Comparing CT and RT-PCR Viral Nucleic Acid Testing.AJR Am J Roentgenol 2020; 215 (4): 834-8.

64. Tofighi S, Najafi S, Johnston SK, Gholamrezanezhad A. Low-dose CT in COVID-19 outbreak: radiation safety, image wisely, and image gently pledge. Emerg Radiol 2020; 27 (6): 601-5.

65. The World Health Organization. COVID-19 Clinical management. 2021. https://www.who.int/publications/i/item/WHO-2019-nCoV-clinical-2021-1 (Accessed March 2021).

66. Mockel M, Corman VM, Stegemann MS, et al. SARS-CoV-2 Antigen Rapid Immunoassay for Diagnosis of COVID-19 in the Emergency Department.Biomarkers 2021: 1-13.

67. Wake RM, Morgan M, Choi J, Winn S. Reducing nosocomial transmission of COVID-19: implementation of a COVID-19 triage system. Clin Med (Lond) 2020; 20 (5): e141-e5.

68. The US Food and Drug Administration. COVID-19 Test Uses: FAQs on Testing for SARS-CoV2. 2021. https://www.fda.gov/medical-devices/coronavirus-covid-19-and-medical-devices/covid-19-test-usesfaqs-testing-sars-cov-2 (accessed March 2021 2021).

69. Huang J, Zheng L, Li Z, et al. Kinetics of SARS-CoV-2 positivity of infected and recovered patients from a single center. Scientific Reports 2020; 10 (1): 18629.

70. Kim SM, Hwang YJ, Kwak Y. Prolonged SARS-CoV-2 detection and reversed RT-PCR results in mild or asymptomatic patients. Infect Dis (Lond) 2021; 53 (1): 31-7.

71. Landi F, Gremese E, Rota E, et al. Positive RT-PCR nasopharyngeal swab in patients recovered from COVID-19 disease: When does quarantine really end? The Journal of infection 2020; 81 (6): e1-e3.

72. Pray IW, Ford L, Cole D, et al. Performance of an Antigen-Based Test for Asymptomatic and Symptomatic SARS-CoV-2 Testing at Two University Campuses - Wisconsin, September-October 2020. MMWR Morb Mortal Wkly Rep 2021; 69 (5152): 1642-7.

73. Carpenter CR, Mudd PA, West CP, Wilber E, Wilber ST. Diagnosing COVID-19 in the Emergency Department: A Scoping Review of Clinical Examinations, Laboratory Tests, Imaging Accuracy, and Biases.Academic emergency medicine : official journal of the Society for Academic Emergency Medicine 2020; 27 (8): 653-70.

74. The European Centre for Disease Prevention and Control. Novel coronavirus (SARS-CoV-2): Discharge criteria for confirmed COVID-19 cases - When is it safe to discharge COVID-19 cases from the hospital or end home isolation? . 2020. https://www.ecdc.europa.eu/sites/default/files/documents/COVID-19Discharge-criteria.pdf (accessed February 2021 2021).

75. The US Centers for Disease Prevention and Control. Discontinuation of Transmission-Based Precautions and Disposition of Patients with SARS-CoV-2 Infection in Healthcare Settings. 2021. https://www.cdc.gov/coronavirus/2019-ncov/hcp/disposition-hospitalized-patients.html (Accessed March 2021).

76. Robert Koch Institut. COVID-19: Release Criteria from Isolation. 2021. https://www.rki.de/DE/Content/InfAZ/N/Neuartiges_Coronavirus/Entlassmanagement.html;jsessionid=5BED1087E0E0E4BCC211959326363099.internet061?nn=13490888 (Accessed March 2021). 
77. Elsayed SM, Reddy MK, Murthy PM, et al. The Possibility and Cause of Relapse After Previously Recovering From COVID-19: A Systematic Review.Cureus 2020; 12 (9): e10264-e.

78. Nakajima Y, Ogai A, Furukawa K, et al. Prolonged viral shedding of SARS-CoV-2 in an immunocompromised patient. Journal of Infection and Chemotherapy 2021; 27 (2): 387-9.

79. Rao SN, Manissero D, Steele VR, Pareja J. A Systematic Review of the Clinical Utility of Cycle Threshold Values in the Context of COVID-19.Infect Dis Ther 2020; 9 (3): 573-86.

80. Piralla A, Ricchi M, Cusi MG, et al. Residual SARS-CoV-2 RNA in nasal swabs of convalescent COVID19 patients: Is prolonged quarantine always justified? International Journal of Infectious Diseases2021; 102 : 299-302.

81. Bielecki M, Patel D, Hinkelbein J, et al. Air travel and COVID-19 prevention in the pandemic and peri-pandemic period: A narrative review. Travel Med Infect Dis 2021; 39 : 101915.

82. Lohse S, Pfuhl T, Berko-Gottel B, et al. Pooling of samples for testing for SARS-CoV-2 in asymptomatic people. Lancet Infect Dis2020; 20 (11): 1231-2.

83. Frnda J, Durica M. On Pilot Massive COVID-19 Testing by Antigen Tests in Europe. Case Study: Slovakia. Infect Dis Rep 2021;13 (1): 45-57.

84. Scohy A, Anantharajah A, Bodeus M, Kabamba-Mukadi B, Verroken A, Rodriguez-Villalobos H. Low performance of rapid antigen detection test as frontline testing for COVID-19 diagnosis. J Clin Virol 2020;129 : 104455 .

85. Control ECfDPa. Infection prevention and control and preparedness for COVID-19 in healthcare settings. 2020 (accessed February 2021 2021).

86. Gholami M, Fawad I, Shadan S, et al. COVID-19 and healthcare workers: A systematic review and meta-analysis. International Journal of Infectious Diseases 2021; 104 : 335-46.

87. DiFiori JP, Green G, Meeuwisse W, Putukian M, Solomon GS, Sills A. Return to sport for North American professional sport leagues in the context of COVID-19. British Journal of Sports Medicine 2020: bjsports-2020-103227.

88. Mooney M, Perera NKP, Broderick C, et al. A deep dive into testing and management of COVID-19 for Australian high performance and professional sport. J Sci Med Sport 2020; 23 (7): 664-9.

89. Pedersen L, Lindberg J, Lind RR, Rasmusen H. Reopening elite sport during the COVID-19 pandemic: Experiences from a controlled return to elite football in Denmark. Scand J Med Sci Sports 2021.

90. Viner RM, Mytton OT, Bonell C, et al. Susceptibility to SARS-CoV-2 Infection Among Children and Adolescents Compared With Adults: A Systematic Review and Meta-analysis. JAMA Pediatrics 2021;175 (2): 143-56.

91. Otte im Kampe E, Lehfeld A-S, Buda S, Buchholz U, Haas W. Surveillance of COVID-19 school outbreaks, Germany, March to August 2020. Eurosurveillance 2020; 25 (38): 2001645.

92. Voysey M, Clemens SAC, Madhi SA, et al. Safety and efficacy of the ChAdOx1 nCoV-19 vaccine (AZD1222) against SARS-CoV-2: an interim analysis of four randomised controlled trials in Brazil, South Africa, and the UK. The Lancet 2021; 397 (10269): 99-111.

93. Polack FP, Thomas SJ, Kitchin N, et al. Safety and Efficacy of the BNT162b2 mRNA Covid-19 Vaccine. New England Journal of Medicine2020; 383 (27): 2603-15.

94. The New York Times. Coronavirus Vaccine Tracker. 2021. https://www.nytimes.com/interactive/2020/science/coronavirus vaccine-tracker.html (Accessed March 2021). 
95. Hodgson SH, Mansatta K, Mallett G, Harris V, Emary KRW, Pollard AJ. What defines an efficacious COVID-19 vaccine? A review of the challenges assessing the clinical efficacy of vaccines against SARS-CoV2. The Lancet Infectious Diseases 2021; 21 (2): e26-e35.

96. The World Health Organization. Guidelines on clinical evaluation of vaccine: regulatory expectations. 2016. https://www.who.int/biologicals/expert_committee/Clinical_changes_IK_final.pdf (Accessed February 2021).

97. Manisty C, Otter AD, Treibel TA, et al. Antibody response to first BNT162b2 dose in previously SARSCoV-2-infected individuals.Lancet 2021.

98. Muller L, Ostermann PN, Walker A, et al. Sensitivity of anti-SARS-CoV-2 serological assays in a highprevalence setting.Eur J Clin Microbiol Infect Dis 2021.

99. Kohmer N, Westhaus S, Ruhl C, Ciesek S, Rabenau HF. Brief clinical evaluation of six high-throughput SARS-CoV-2 IgG antibody assays.J Clin Virol 2020; 129 : 104480.

100. Ozcurumez MK, Ambrosch A, Frey O, et al. SARS-CoV-2 antibody testing-questions to be asked. $J$ Allergy Clin Immunol 2020;146 (1): 35-43.

101. The US Centers for Disease Prevention and Control. Genomic surveillance for SARS-CoV-2. 2021. https://www.cdc.gov/coronavirus/2019-ncov/cases-updates/variant-surveillance.html (Accessed February 2021).

102. Wu K, Werner AP, Moliva JI, et al. mRNA-1273 vaccine induces neutralizing antibodies against spike mutants from global SARS-CoV-2 variants. bioRxiv 2021: 2021.01.25.427948.

103. US Food and Drug Administration. Genetic Variants of SARS-CoV-2 May Lead to False Negative Results with Molecular Tests for Detection of SARS-CoV-2 - Letter to Clinical Laboratory Staff and Health Care Providers. 2021. https://www.fda.gov/medical-devices/letters-health-care-providers/genetic-variantssars-cov-2-may-lead-false-negative-results-molecular-tests-detection-sars-cov-2 (Accessed February 2021).

104. Buchta C, Görzer I, Chiba P, et al. Variability of cycle threshold values in an external quality assessment scheme for detection of the SARS-CoV-2 virus genome by RT-PCR. Clinical Chemistry and Laboratory Medicine (CCLM) 2020.

105. Javalkote VS, Kancharla N, Bhadra B, et al. CRISPR-based assays for rapid detection of SARS-CoV-2. Methods 2020.

106. Xiong E, Jiang L, Tian T, et al. Simultaneous Dual-Gene Diagnosis of SARS-CoV-2 Based on CRISPR/Cas9-Mediated Lateral Flow Assay.Angew Chem Int Ed Engl 2020. 